\title{
Experimental investigation on rheological property of bitumen influenced by preheating process
}

\author{
Xuemei Zhang \& Inge Hoff \\ Department of Civil and Environmental Engineering, Norwegian University of Science and Technology, \\ Trondheim, Norway
}

\begin{abstract}
Bitumen samples for laboratory tests are normally preheated at moderate temperature. However, the preheating process is suspect of influencing the aging process and bitumen property. This research aims to investigate the rheological property of bitumen influenced by preheating process. For this purpose, the matrix bitumen and bitumen aged by ThinFilm Oven Test (TFOT) were preheated for seven cycles, respectively. Dynamic Shear Rheometer (DSR) test was conducted to explore the influence of preheating process on rheological properties of bitumen. The results showed that preheating process shortened the differences in rheological properties between matrix and aged bitumen over preheating cycle. The effect of preheating process on bitumen could be divided into three stages: rapid change stage, stable stage, and aged stage. The rapid change stage, in which bitumen volatilizes rapidly resulting in apparent changes in rheological properties, typically occurs in the first two preheating cycles. The stable stage in which bitumen remains few changes with preheating cycle lasts for a longer period (3-5 preheating cycles). The aged stage, in which excessive heating time is applied on bitumen resulting in the aging of bitumen, usually starts from the sixth preheating cycle. The consequences of this study provide practitioners with proposals to reduce experimental error for bituminous tests by avoiding preheating bitumen more than six times.
\end{abstract}

Keywords: Bitumen, preheating process, rheological property, three stages

\section{INTRODUCTION}

Bitumen as a viscoelastic material plays a significant role in determining the service life of asphalt pavement, although it only accounts for 4-6\% of the gross mass of asphalt concrete (Li et al., 2019; Morova, 2013; Serin et al., 2012). The viscoelastic behaviour of bitumen could be comprehensively characterized by the rheological properties of bitumen. Rheological properties of bitumen primarily include deformation resistance, elastic/viscous response, rutting resistance, and resistance to fatigue cracking, which are of great importance in predicting asphalt pavement distresses (Remisova et al., 2016; Tao et al., 2019).

For laboratory tests, bitumen is inevitably preheated several times for subsequent tests. To be specific, when a barrel of bitumen is obtained from an asphalt factory, a barrel of bitumen should be preheated (up to $100{ }^{\circ} \mathrm{C}$ ) to fluid and taken out to make subsequent test samples according to NS-EN 12594:2014. Thus, bitumen might be heated several times until the bitumen bucket is empty. However, several preheating cycles are suspected of aging bitumen i.e., excessive heating time would promote the volatilization or oxidation of bitumen (Ye et al., 2019). 
In addition, microwave heating and induction heating as the major healing techniques can also be regarded as a heating action to bitumen (Li et al., 2020; Liu et al., 2020; Trigos et al., 2020). Previous studies showed that the temperature of asphalt concrete after microwave heating and induction heating could reach up to $91{ }^{\circ} \mathrm{C}$ and $83{ }^{\circ} \mathrm{C}$, respectively (Gonzalez et al., 2018; Sun et al., 2020). These temperatures are beyond the softening point of bitumen. Therefore, the healing process performed on asphalt concrete could be regarded as the preheating process.

Therefore, the influence of the preheating process on the rheological properties of bitumen should be paid more attention. For this purpose, two kinds of bitumen, matrix bitumen and aged bitumen by Thin Film Oven Test (TFOT) aging, were preheated for seven cycles, respectively. The rheological properties of both matrix bitumen and aged bitumen were investigated by Dynamic Shear Rheometer (DSR) test. The conclusions obtained from the research could give some suggestions on reducing experimental error for bituminous tests.

\section{MATERIALS AND METHODS}

\subsection{Materials}

The matrix bitumen applied in this research was obtained from company Veidekke in Norway. Aged bitumen was aged by thin film oven test (TFOT) according to NS-EN 12607$2: 2014$. The basic properties of bitumen are shown in Table 1 . The penetration of bitumen is tested according to NS-EN 1426:2015 standard. The mass loss caused by TFOT aging is calculated according to formula 1.

$$
\text { Mass loss }=\frac{\mathrm{m}_{\mathrm{A}}-\mathrm{m}_{\mathrm{U}}}{\mathrm{m}_{\mathrm{U}}} \times 100 \%
$$

Where $\mathrm{m}_{\mathrm{U}}$ is the mass of matrix bitumen, $\mathrm{m}_{\mathrm{A}}$ is the mass of TFOT aged bitumen.

Table 1. Basic properties of bitumen.

\begin{tabular}{llll}
\hline & \multicolumn{2}{l}{ Penetration $[\mathrm{mm}]$} & \\
\cline { 2 - 3 } $\begin{array}{l}\text { The number of } \\
\text { preheating cycles }\end{array}$ & Matrix bitumen & TFOT aged bitumen & Mass loss [\%] \\
\hline 1 & 7.71 & 5.44 & \\
2 & 7.66 & 5.72 & -0.05 \\
3 & 7.41 & 5.79 & -0.03 \\
4 & 7.52 & 5.85 & -0.02 \\
5 & 6.99 & 5.40 & 0 \\
6 & 7.01 & 5.52 & -0.02 \\
7 & 7.05 & 5.75 & -0.02 \\
\hline
\end{tabular}

\subsection{Methodology}

\subsubsection{Thin film oven test aging}

Thin-film oven test aging (TFOT) test was conducted to simulate the short-term aging of bitumen during storage and transportation. The test parameters are shown in Table 2.

\subsubsection{Dynamic shear rheometer}

In this research, rheological behaviours of bitumen were measured using Dynamic Shear Rheometer (DSR). The test parameters are presented in Table 3 . The temperature is chosen as $10^{\circ} \mathrm{C}$ and $20^{\circ} \mathrm{C}$, which is reasonable and practical temperature condition for bitumen in reality. 
Table 2. Test parameters.

\begin{tabular}{llll}
\hline Test parameters & Value & Test parameters & Value \\
\hline Diameter of metallic pan & $140 \quad 1 \mathrm{~mm}$ & Oven temperature & $163^{\circ} \mathrm{C}$ \\
The mass of each sample & $50 \mathrm{~g}$ & Aging/heating time & $5 \mathrm{~h}$ \\
Thickness of bitumen film & $3.2 \mathrm{~mm}$ & Cooling time & $2 \mathrm{~h}$ \\
\hline
\end{tabular}

Table 3. Parameters value.

\begin{tabular}{ll}
\hline Setting & Value for rheological property \\
\hline Angular frequency & $10 \mathrm{rad} / \mathrm{s}$ \\
Amplitude gamma & $0.5 \%$ \\
Temperature range & $10{ }^{\circ} \mathrm{C}, 20^{\circ} \mathrm{C}$ \\
Diameter of the plate & $8 \mathrm{~mm}$ \\
Sample thickness & $2 \mathrm{~mm}$ \\
\hline
\end{tabular}

\subsubsection{Preheating procedure}

In terms of the preheating procedure, a barrel of bitumen is heated at $90{ }^{\circ} \mathrm{C}$ for 1.5 hours in accordance with the NS-EN 12594:2014 standard so that bitumen was liquid enough for conducting subsequent tests. This procedure is called the preheating of bitumen.

The flow diagram of the preheating cycle for matrix bitumen and aged bitumen is presented in Figure 1. Firstly, a bucket of bitumen is preheated $n$ times and then placed in a metallic plate for TFOT test. After the preheating cycles or TFOT aging, the rheological properties of both matrix bitumen and aged bitumen are studied by DSR tests. Meanwhile, U-n indicates matrix bitumen after $\mathrm{n}$ preheating cycles, A-n indicates TFOT aged bitumen after $\mathrm{n}$ preheating cycles. Regarding the number of preheating cycles, both matrix bitumen and TFOT aged bitumen are preheated for $1,2,3,4,5,6$, and 7 cycles, respectively.

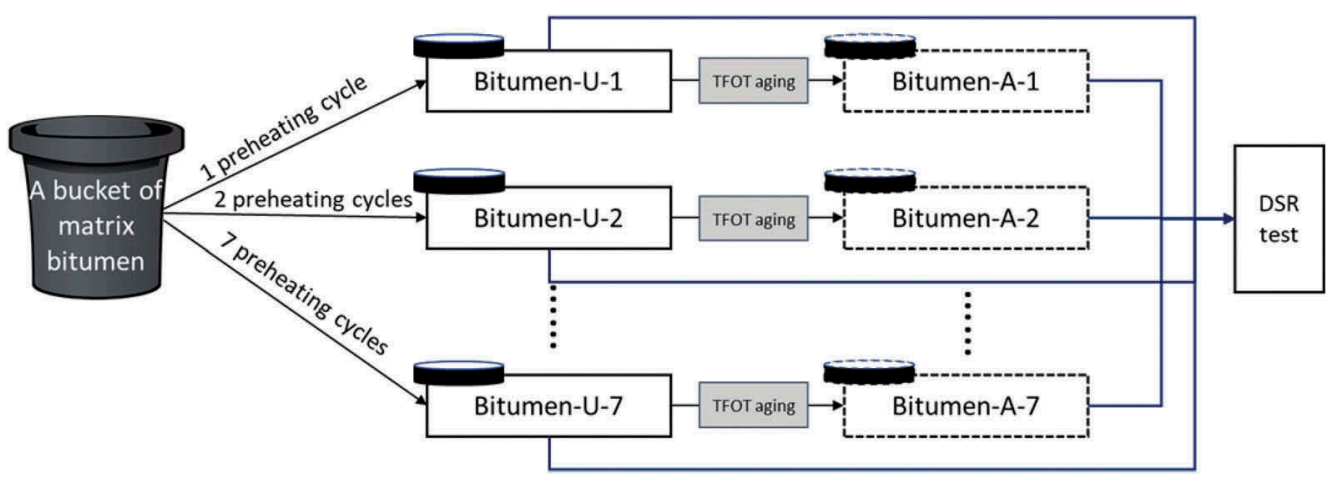

Figure 1. The flowchart of preheating cycle for matrix bitumen and aged bitumen. 


\section{RESULTS AND DISCUSSIONS}

\subsection{Complex modulus}

The complex modulus $\left(\mathrm{G}^{*}\right)$ is defined as the total resistance to deformation of bitumen, and higher complex modulus indicates better resistance to deformation (Farias et al., 2016). Figure 2 shows the complex modulus of matrix and aged bitumen over preheating cycle at $10{ }^{\circ} \mathrm{C}$ and $20^{\circ} \mathrm{C}$. Regarding matrix bitumen, U-3 and U-4 performed the highest values of complex modulus, and the complex modulus of U-1 was the smallest. These phenomena show little correlation in complex modulus between matrix bitumen and preheating cycle. However, in terms of aged bitumen, A-2 and A-1 have the biggest complex modulus, and A-7 had the smallest complex modulus. Comparing matrix bitumen and aged bitumen, the preheating process changed the order of complex modulus of bitumen samples.
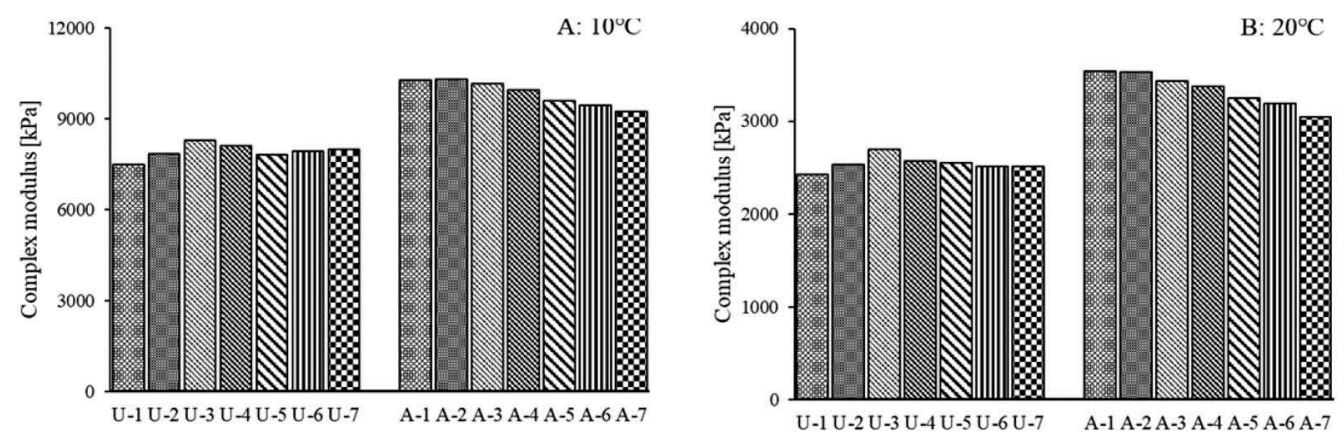

Figure 2. The complex modulus of samples versus preheating cycle.

In order to evaluate the complex modulus of bitumen influenced by the preheating cycle, the change of complex modulus between aged bitumen and matrix bitumen under the same preheating cycle is calculated and shown in Figure 3. The change of complex modulus decreased with the increasing preheating cycle. This phenomenon means that the preheating process causes a significant impact on bituminous resistance to deformation, and more preheating cycles would result in smaller differences in complex modulus between matrix bitumen and aged bitumen. Bitumen samples after 1 and 2 preheating cycles showed relatively higher values of complex modulus than other samples, which means the deformation resistance of bitumen is vulnerable to preheating procedure at the beginning (1-2 preheating cycles). Bitumen samples after 3, 4, 5, and 6 preheating cycles showed similar values of the change of complex modulus, which indicates these samples are relatively stable. Besides, bitumen after 7 preheating cycles had the smallest change of complex modulus than middle four samples. This conclusion demonstrates that the matrix bitumen after 7 preheating cycles is slightly aged so that the difference in complex modulus between matrix and aged bitumen is relatively small.

\subsection{Phase angle}

Phase angle $(\delta)$ is defined as the ratio of the loss to the storage components of the complex modulus, the phase angle of bitumen is between $0^{\circ}$ to $90^{\circ}$, a higher $\delta$ indicates better viscous response and worse elastic response of bitumen (He et al., 2019). Figure 4A and Figure 4B show the results of the phase angle of samples at $10{ }^{\circ} \mathrm{C}$ and $20{ }^{\circ} \mathrm{C}$ after different preheating cycles, respectively. U-6 and U-4 have relatively bigger phase angles among matrix samples. U-1 has the smallest $\delta$ among matrix samples. Regarding aged bitumen, A-6 and A-7 have the 


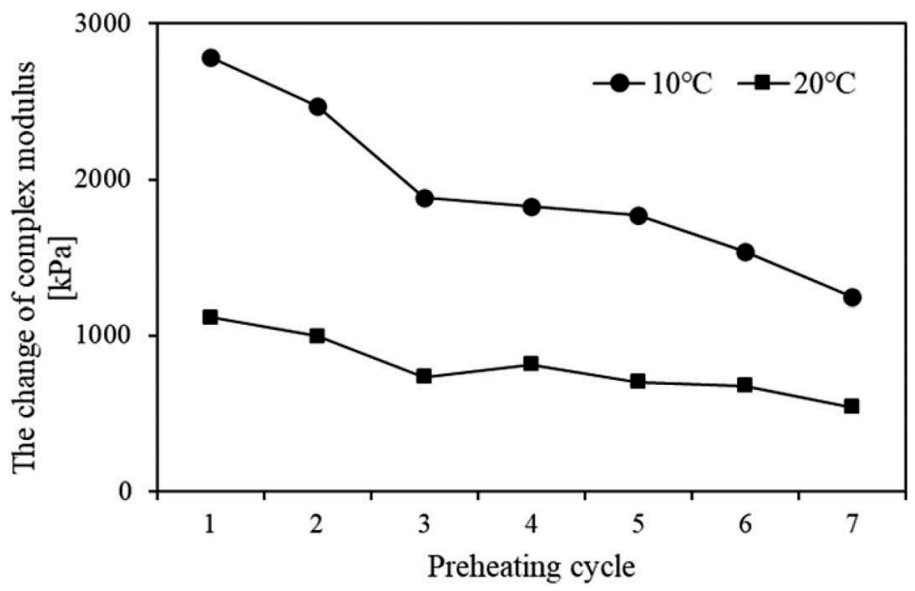

Figure 3. The changes in complex modulus between matrix and aged bitumen versus preheating cycle.
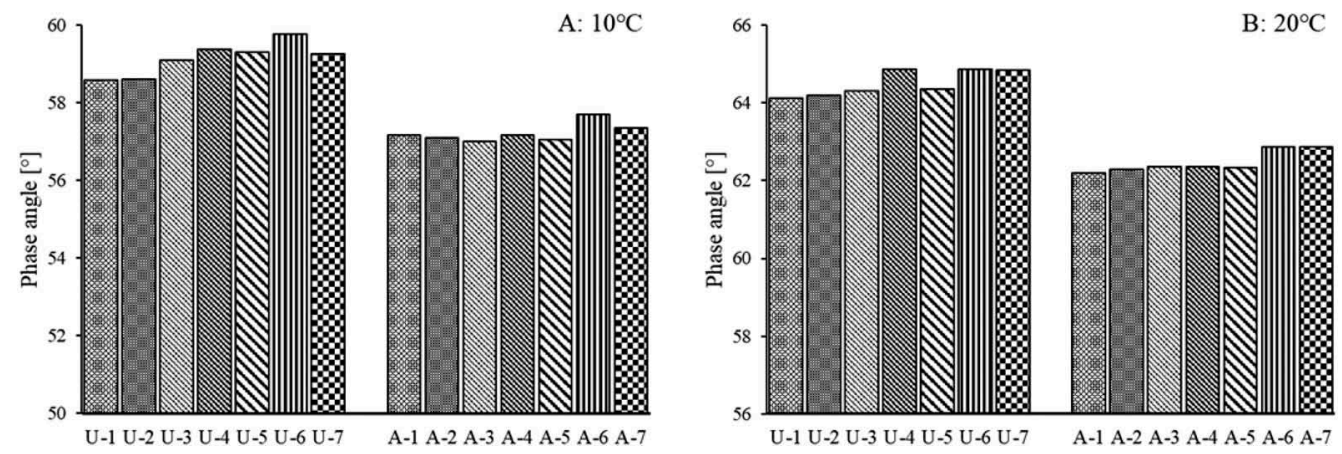

Figure 4. The phase angle of samples versus preheating cycle.

biggest phase angle, and the phase angle of A-3 and A-5 is relatively small. These results indicate that the preheating process has limited influence on the viscoelastic response of matrix bitumen but affects the short-term aging on bitumen.

The contrast of matrix bitumen and aged bitumen is estimated, which results in the difference of phase angle between matrix bitumen and aged bitumen over preheating cycle shown in Figure 5. The changed value of phase angle of all samples is below $3^{\circ}$. Phase angle showed a different changing trend over the preheating cycle compared to complex modulus. The phase angle of bitumen decreased before 4 preheating cycles and remained few changes afterward. Meanwhile, the phase angles of bitumen samples after 1 and 2 preheating cycles were the biggest among all samples. These facts indicate that bitumen is intensely affected at the beginning (1-2 preheating cycles) and then keeps stable after 4 preheating cycles. 


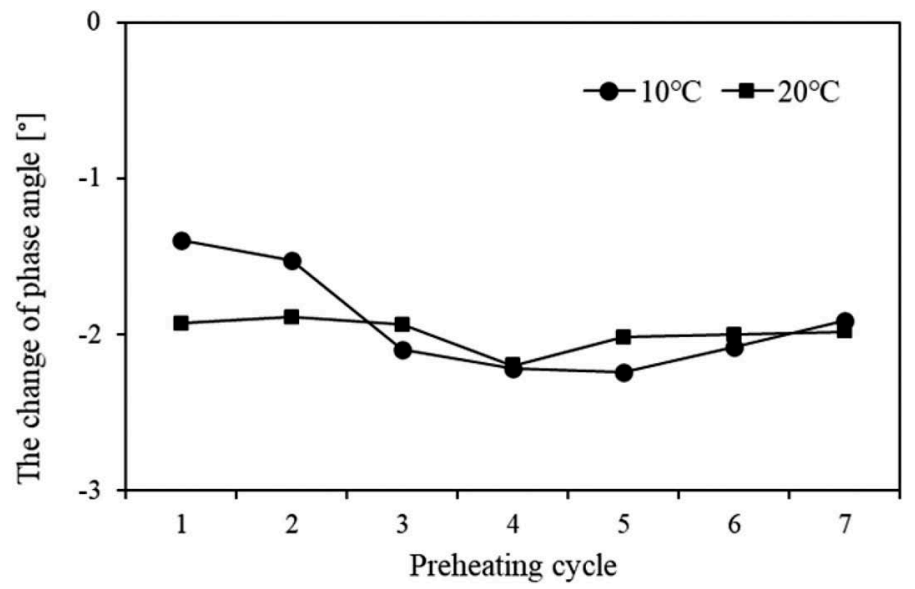

Figure 5. The changes in phase angle between matrix and aged bitumen versus preheating cycle.

\subsection{Rutting factor}

Rutting factor is defined as the ratio of complex modulus to $\sin \delta$. It reflects the rutting resistance of bitumen. A higher rutting factor indicates better performance to resist rutting/permanent deformation (Sedaghat et al., 2020). Figure 6A and Figure 6B show the result of the rutting factor of samples at $10{ }^{\circ} \mathrm{C}$ and $20^{\circ} \mathrm{C}$ after different preheating cycles, respectively. U-3 and U-4 had higher rutting factors, and U-1 and U-5 had smaller rutting factors among all matrix bitumen samples. These results indicate little correlation between rutting factor of matrix bitumen and preheating cycle. For aged bitumen, A-2 and A-1 had the higher rutting factors, and A-7 and A-6 had the smaller rutting factors. These data indicate that matrix bitumen and aged bitumen perform differently to resist rutting under different preheating cycles. Based on the different orders of samples' rutting factor between matrix and aged bitumen, preheating cycles might affect the degree of TFOT aging on bitumen.
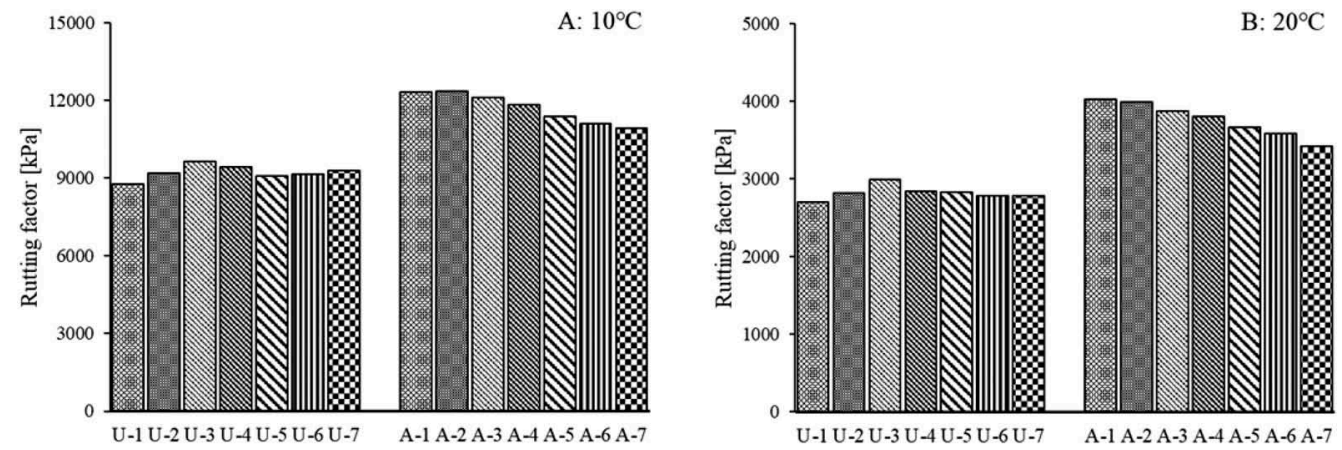

Figure 6. The rutting factor of samples versus preheating cycle. 
In order to study the effect of preheating cycle on the rutting factor of bitumen, the changes in rutting factor between matrix and aged bitumen after different preheating cycles are calculated and shown in Figure 7. The changes in rutting factor of bitumen samples after 1 and 2 preheating cycles were relatively noticeable; samples after 3,4 , or 5 preheating cycles showed similar differences in rutting factor; sample after 7 preheating cycles had the smallest change in rutting factor. These facts show that the change in rutting resistance of bitumen decreased with the increase of preheating cycles. Besides, bitumen is more stable when the number of preheating cycles is in the range of 3 to 5 . Due to the instability of samples, the difference of rutting factor of sample- 1 and sample- 2 is apparent. While after 6 or 7 preheating cycles, the bitumen is already aged to some extent so that the difference in rutting factor between matrix and aged bitumen is relatively small.

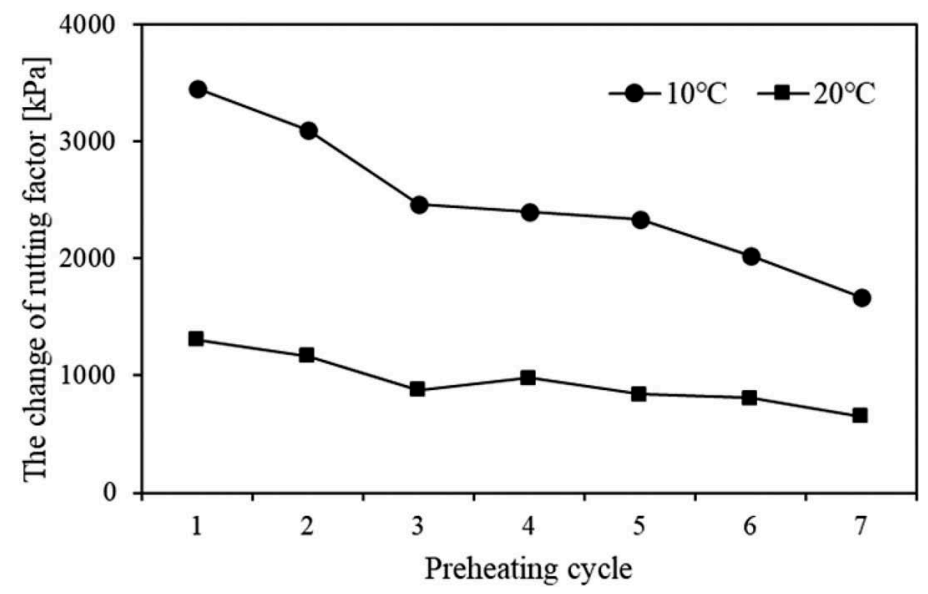

Figure 7. The change in rutting factor between matrix and aged bitumen versus preheating cycle.

\subsection{Fatigue factor}

Fatigue factor is defined as the product of complex modulus and $\sin \delta$, it is used to evaluate the performance against fatigue distress of bitumen. A higher fatigue factor would contribute to a worse ability to resist fatigue cracking (Ameri et al., 2018). Figure 8 shows the fatigue factor of samples at $10{ }^{\circ} \mathrm{C}$ and $20{ }^{\circ} \mathrm{C}$ after different preheating cycles. Regarding the matrix bitumen, U-1 has the lowest fatigue factor, U-3 has the highest fatigue factor. However, in terms of aged bitumen, A-2 has the biggest fatigue factor, followed by A-1, and A-7 has the smallest fatigue factor. These facts indicate that the preheating procedure has little impact on the ability to resist fatigue cracking of matrix bitumen, while it could influence the evolution of bitumen aging leading to the changed order in fatigue factor of aged bitumen.

How preheating procedure influences the fatigue factor is estimated by comparing the fatigue factor between matrix and aged bitumen after the same preheating cycle, which is presented in Figure 9. The change in fatigue factor of samples decreased over preheating cycle, which indicates that more preheating cycles would shorten the differences in fatigue factor between matrix bitumen and aged bitumen. As seen from Figure 9, bitumen samples after 1 and 2 preheating cycles showed higher values in the change of fatigue factor than other samples, which indicates that bitumen is easily influenced with a severe degree after one or two 

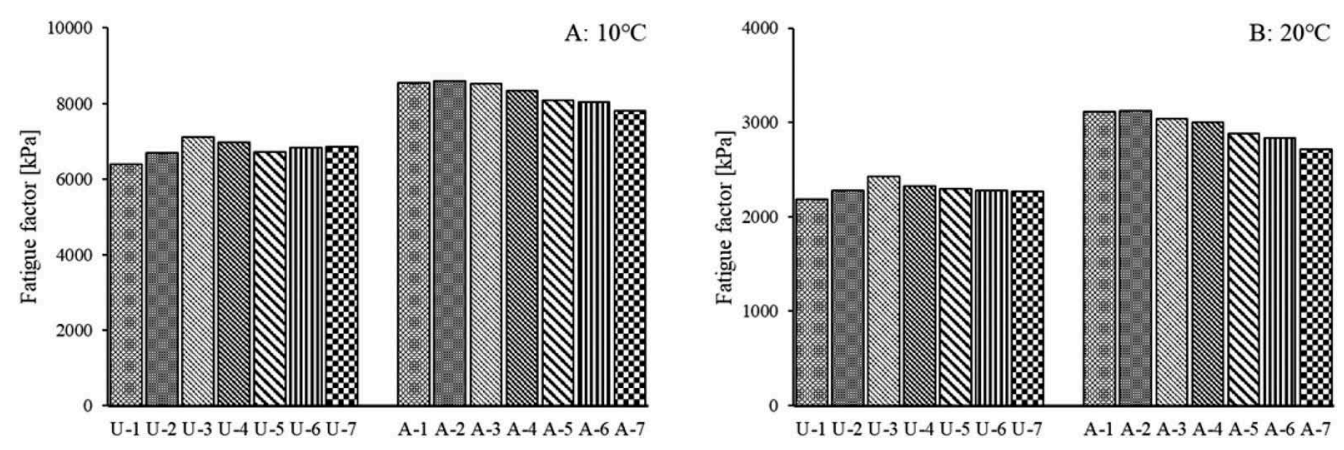

Figure 8. The fatigue factor of samples versus preheating cycle.

preheating cycles. However, after 3, 4, 5, and 6 preheating cycles, the samples showed similar changes in fatigue factor, which indicates that the sample after 3, 4, 5, and 6 preheating cycles have a relatively stable state. Finally, the bitumen with seven preheating cycles had the smallest difference in fatigue factor, which shows that bitumen is already aged to a certain extent so that the difference of matrix and aged bitumen is previously smaller than that after fewer preheating cycles.

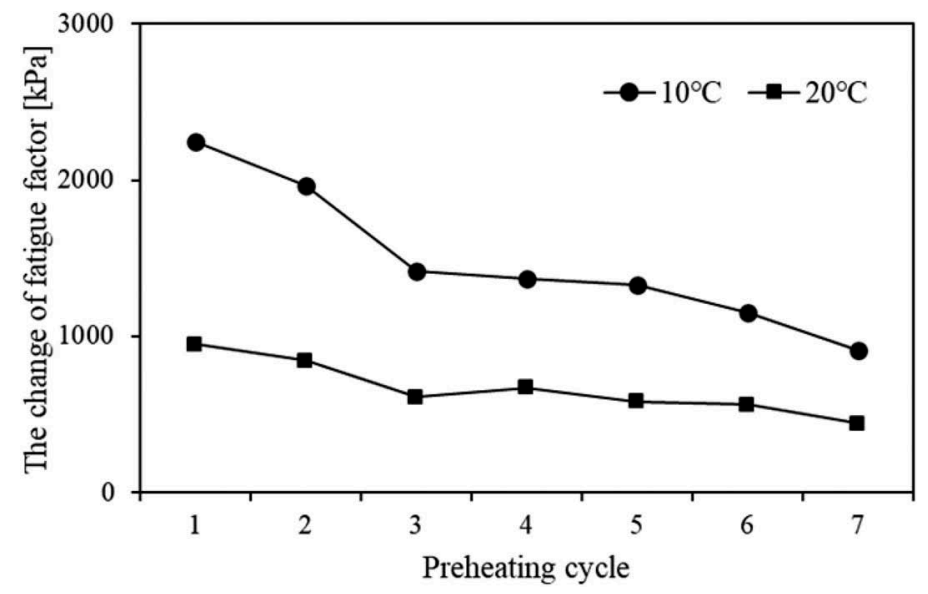

Figure 9. The change in fatigue factor between matrix and aged bitumen versus preheating cycle.

\section{CONCLUSIONS}

For laboratory tests, bitumen is generally preheated at moderate temperature to fluid for the following tests. While the preheating process would influence bitumen rheological properties and the outcome of the following tests. Therefore, this research studied the effect of preheating process on the rheological properties of matrix bitumen and TFOT aged bitumen. The obtained conclusions were summarized as follows.

The preheating process had a limited impact on the rheological properties of matrix bitumen, whereas the preheating process has a significant influence on the short-term aging on bitumen. The difference in rheological properties between matrix bitumen and aged bitumen decreased over preheating cycle. There are three stages during preheating process, i.e., rapid change stage, stable stage, and aged stage. The rapid change stage normally occurs in the first two preheating cycles since bitumen is the most unstable and easily volatilized at the beginning 
resulting in apparent differences in rheological properties. The stable stage in which the bitumen remains few changes in rheological properties lasts for a longer period ( 3 - 5 preheating cycles). The aged stage, in which bitumen is slightly aged resulting in small changes in rheological parameters, normally starts from the sixth preheating cycle.

The conclusions could provide engineers with proposals on how to reduce experimental error for bituminous tests. For example, it is better not to preheat the bitumen sample more than six times to avoid bitumen aging, which could affect the subsequent test results.

\section{DECLARATION OF INTERESTS}

The authors declare that they have no known competing financial interests or personal relationships that could have appeared to influence the work reported in this paper.

\section{REFERENCES}

Ameri, M., Mansourkhaki, A., Daryaee, D., 2018. Evaluation of fatigue behavior of high reclaimed asphalt binder mixes modified with rejuvenator and softer bitumen. Constr Build Mater 191, 702-712.

Farias, L.G.A.T., Leitinho, J.L., Amoni, B.D.C., Bastos, J.B.S., Soares, J.B., Soares, S.D.A., de Sant'Ana, H.B., 2016. Effects of nanoclay and nanocomposites on bitumen rheological properties. Constr Build Mater 125, 873-883.

Gonzalez, A., Norambuena-Contreras, J., Storey, L., Schlangen, E., 2018. Self-healing properties of recycled asphalt mixtures containing metal waste: An approach through microwave radiation heating. J Environ Manage 214, 242-251.

He, R., Zheng, S.N., Chen, H.X., Kuang, D.L., 2019. Investigation of the physical and rheological properties of Trinidad lake asphalt modified bitumen. Constr Build Mater 203, 734-739.

Li, H.C., Yu, J.Y., Wu, S.P., Liu, Q.T., Wu, Y.Q., Xu, H.Q., Li, Y.Y., 2020. Effect of moisture conditioning on mechanical and healing properties of inductive asphalt concrete. Constr Build Mater 241.

Li, Y.Y., Wu, S.P., Liu, Q.T., Nie, S., Li, H.C., Dai, Y., Pang, L., Li, C.M., Zhang, A.M., 2019. Field evaluation of LDHs effect on the aging resistance of asphalt concrete after four years of road service. Constr Build Mater 208, 192-203.

Liu, K., Dai, D.L., Fu, C.L., Li, W.H., Li, S.Q., 2020. Induction heating of asphalt mixtures with waste steel shavings. Constr Build Mater 234.

Morova, N., 2013. Investigation of usability of basalt fibers in hot mix asphalt concrete. Constr Build Mater 47, 175-180.

Remisova, E., Zatkalikova, V., Schlosser, F., 2016. Study of Rheological Properties of Bituminous Binders in Middle and High Temperatures. Civ Environ Eng 12(1), 13-20.

Sedaghat, E., Taherrian, R., Hosseini, S.A., Mousavi, S.M., 2020. Rheological properties of bitumen containing nanoclay and organic warm-mix asphalt additives. Constr Build Mater 243.

Serin, S., Morova, N., Saltan, M., Terzi, S., 2012. Investigation of usability of steel fibers in asphalt concrete mixtures. Constr Build Mater 36, 238-244.

Sun, G.Q., Hu, M.J., Sun, D.Q., Deng, Y., Ma, J.M., Lu, T., 2020. Temperature induced self-healing capability transition phenomenon of bitumens. Fuel 263.

Tao, G.Y., Xiao, Y., Yang, L.F., Cui, P.D., Kong, D.Z., Xue, Y.J., 2019. Characteristics of steel slag filler and its influence on rheological properties of asphalt mortar. Constr Build Mater 201, 439-446.

Trigos, L., Gallego, J., Escavy, J.I., 2020. Heating potential of aggregates in asphalt mixtures exposed to microwaves radiation. Constr Build Mater 230.

Ye, W.L., Jiang, W., Li, P.F., Yuan, D.D., Shan, J.H., Xiao, J.J., 2019. Analysis of mechanism and time-temperature equivalent effects of asphalt binder in short-term aging. Constr Build Mater 215, $823-838$. 